\title{
In silico comparison of pharmacokinetic properties of three extended half-life factor IX concentrates
}

\author{
Tim Preijers $^{1} \cdot$ Laura Bukkems $^{1} \cdot$ Max van Spengler ${ }^{1} \cdot$ Frank Leebeek $^{2} \cdot$ Marjon Cnossen $^{3} \cdot$ Ron Mathôt $^{1}$ (I)
}

Received: 20 October 2020 / Accepted: 11 February 2021 / Published online: 24 February 2021

(C) The Author(s) 2021

\begin{abstract}
Purpose Pharmacokinetic (PK) differences between the extended half-life (EHL) factor IX (FIX) concentrates for hemophilia B exist, which may influence hemostatic efficacy of replacement therapy in patients. Therefore, we aimed to evaluate the PK properties of three EHL-FIX concentrates and compare them to a standard half-life (SHL) recombinant FIX (rFIX) concentrate. Methods Activity-time profiles of PEGylated FIX (N9-GP), FIX linked with human albumin (rIX-FP), FIX coupled to human IgG1 Fc-domain (rFIXFc), and SHL rFIX were simulated for 10,000 patients during steady-state dosing of $40 \mathrm{IU} / \mathrm{kg}$ once weekly (EHL-FIX) and biweekly (rFIX) using published concentrate specific population PK models.

Results Half-lives were respectively 80, 104, and $82 \mathrm{~h}$ for N9-GP, rIX-FP, and rFIXFc versus $22 \mathrm{~h}$ for rFIX. Between the EHL concentrates, exposure was different with area under the curve (AUC) values of 78.5, 49.6, and $12.1 \mathrm{IU} / \mathrm{h} / \mathrm{mL}$ and time above FIX target values of $0.10 \mathrm{IU} / \mathrm{mL}$ of 168,168 , and $36 \mathrm{~h}$ for N9-GP, rIX-FP, and rFIXFc, respectively. N9-GP produced the highest median in vivo recovery value $(1.70 \mathrm{IU} / \mathrm{dL}$ per IU/kg) compared with $1.18,1.00$, and $1.05 \mathrm{IU} / \mathrm{dL}$ per IU $/ \mathrm{kg}$ for rIX-FP, rFIXFc, and rFIX, respectively.

Conclusions When comparing EHL products, not only half-life but also exposure must be considered. In addition, variation in extravascular distribution of the FIX concentrates must be taken into account. This study provides insight into the different PK properties of these concentrates and may aid in determination of dosing regimens of EHL-FIX concentrates in real-life.
\end{abstract}

Keywords Factor IX $\cdot$ Hemophilia B $\cdot$ Pharmacokinetics $\cdot$ Half-life $\cdot$ Comparative study

\section{Introduction}

Hemophilia B patients are characterized by a deficiency of coagulation factor IX (FIX) resulting in bleeding, typically in joints and muscles [1]. It has been demonstrated that patients with moderate and mild hemophilia — defined as a baseline FIX level of $>0.01 \mathrm{IU} / \mathrm{mL}$ and $>0.05 \mathrm{IU} / \mathrm{mL}$,

Marjon Cnossen and Ron Mathôt are both last authors.

Ron Mathôt

r.mathot@amsterdamumc.nl

1 Hospital Pharmacy-Clinical Pharmacology, Amsterdam University Medical Center, Meibergdreef 9, P.O. Box 22660, 1100 DD Amsterdam, Netherlands

2 Department of Hematology, Erasmus MC, University Medical Center Rotterdam, Rotterdam, Netherlands

3 Department of Pediatric Hematology, Erasmus MC-Sophia Children's Hospital, University Medical Center Rotterdam, Rotterdam, Netherlands respectively - experience spontaneous bleeding less frequently and demonstrate delayed development of arthropathy when compared with severe hemophilia patients $(<0.01 \mathrm{IU} / \mathrm{mL})$ [2]. Therefore, traditionally severe hemophilia B patients administrate FIX concentrate prophylactically to maintain FIX trough levels of at least $>0.01 \mathrm{IU} / \mathrm{mL}$ [3]. However, due to inter-individual variation in bleeding tendency the sufficient FIX target level during prophylaxis to prevent bleeding can vary between patients. Some patients do not experience bleeding when trough levels are $<0.01 \mathrm{IU} / \mathrm{mL}$ while others require higher factor trough levels $[4,5]$. In spite of these findings, it has been demonstrated in hemophilia A patients that longer time intervals spent with factor VIII activity levels $>0.01 \mathrm{IU} /$ $\mathrm{mL}$ resulted in lower annualized bleeding rates [6]. Some studies even suggested to aim for higher trough activity levels to prevent bleeds [7]. Therefore, higher FIX trough activity levels may be required for some patients, depending on bleeding tendency, level of physical activity, and joint status [8]. As a result, not only trough FIX activity levels but also area under the activity level versus time curve (AUC) and time spent with 
FIX activity levels above $0.03 \mathrm{IU} / \mathrm{mL}, 0.05 \mathrm{IU} / \mathrm{mL}$, and 0.10 $\mathrm{IU} / \mathrm{mL}$ are expected to be important determinants to predict bleeding risk.

Efforts have been made to modify the pharmacological properties of FIX concentrates in order to extend its terminal half-life and/or augment its in vivo hemostatic function [9, 10]. Currently, three extended half-life (EHL) FIX concentrates are widely available: PEGylated FIX (N9-GP), recombinant FIX linked with recombinant human albumin (rIX-FP), and FIX coupled to the human IgG1 Fc domain (rFIXFc) [11, 12]. Whereas standard half-life (SHL) FIX concentrates are generally administered twice weekly to maintain target FIX trough levels, EHL-FIX concentrates can be administered once weekly or possible even less frequently [13]. One of the greatest advantage of these EHL-FIX concentrates is the reduction in frequency of infusion, especially in patients with difficult venous access. On the contrary, less frequent administration of EHL-FIX concentrates may also result in longer time intervals at relatively low FIX activity levels, which may actually lead to lower hemostatic efficacy especially for patients requiring higher trough levels. For this reason, it is also important to examine the time patients spent above a specified FIX activity level.

Although the EHL-FIX products have been designed to have altered elongating PK properties when compared with SHL-rFIX products, these have not yet been simultaneously compared in a clinical study. A simultaneous comparison between PK properties of the EHL-FIX products can be useful, as the PK properties described in clinical trials are obtained with different dosing regimens making comparison of several PK properties difficult. Furthermore, in the reports of these clinical trials, clinically interesting PK properties such as time spent above a certain factor level are often not presented. Nevertheless, population PK models have been published for the examined concentrates, making evaluation using Monte Carlo simulations possible. Monte Carlo simulations not only allow the comparison of PK parameters in a typical or average patient but also illustrate the associated interpatient variability observed in a patient population. Application of Monte Carlo simulations can be beneficial as costs and exposure of the patient to an intervention are minimized while maximizing similarity with clinical practice. Therefore, the objective of this study was to compare the PK properties of three currently available EHL-FIX concentrates to a widely used SHL-rFIX concentrate using Monte Carlo simulations.

\section{Methods}

Monte Carlo simulations were performed to produce FIX activity levels versus time profiles of three EHL-FIX concentrates N9-GP (Refixia ${ }^{\circledR}$, Novo Nordisk A/S, Denmark), rIX-FP (Idelvion ${ }^{\circledR}$, CSL Behring GmbH, Germany), and rFIXFc (Alprolix ${ }^{\circledR}$, Swedish Orphan Biovitrum AB, Sweden) and one SHL-rFIX concentrate (BeneFIX®, Pfizer, UK) in 10,000 virtual patients [14]. In a Monte Carlo simulation, a population PK model is used to generate individual PK parameters and subsequent FIX levels for each desired time-point. Residual error was not included in the simulated FIX levels. The simulations were performed with NONMEM v7.4.1. using population PK models reported in literature (Table 1) [15-17]. For N9-GP, only a population PK model based on phase 1 trial data was available in literature $[18,19]$. In the phase 1 N9-GP trial, FIX levels were measured using a modified aPTT-based assay with a Trinity auto aPTT reagent (silica-based), while in the phase 3 trials, FIX levels were measured using an aPTT-based one-stage assay with a SynthAFax reagent $[20,21]$. The activity of N9-GP is generally overestimated when a silica-based reagent is used, as applied in the phase 1 trial $[22,23]$. Therefore, updated population PK parameters of N9-GP were generously provided by Novo Nordisk based on data from the phase 3 trials.

$\mathrm{R}$ software (v3.4.3) was used to create the population of 10,000 virtual severe hemophilia B patients [24].

Different age and bodyweight characteristics were assigned to the virtual patients. The ranges of these simulated characteristics were based on the combined age and bodyweight ranges from the studied populations of the population PK models available in literature to avoid extrapolation. Therefore, simulated age and bodyweight ranged from 21 to 65 years and from 57.3 to $90 \mathrm{~kg}$, respectively. The relation between age and weight and distribution of these characteristics was simulated using the tmvtnorm package in R. For reasons of simplicity, PK of the EHL-FIX was only evaluated in severe hemophilia B patients (endogenous baseline level $<0.01 \mathrm{IU} / \mathrm{mL}$ ). Consequently, no endogenous baseline FIX level was simulated for the virtual patients. The population PK model for rIX-FP contained a structural parameter to describe the baseline FIX levels of hemophilia B patients. This parameter was, however, subsequently discarded during the Monte Carlo simulations, as baseline FIX levels were $<0.01$ $\mathrm{IU} / \mathrm{mL}$.

In the simulations, steady-state PK was present in all patients, receiving $40 \mathrm{IU} / \mathrm{kg}$ of EHL-FIX once weekly and 40 IU/kg SHL-rFIX twice-weekly. For each virtual patient, the following PK parameters were calculated: terminal elimination half-life, AUC (from 0 to $168 \mathrm{~h}$ ), maximum FIX activity level, in vivo recovery, and FIX trough activity level. Moreover, the time below and above 0.01, 0.03, 0.05, and $0.10 \mathrm{IU} / \mathrm{mL}$ was calculated. Furthermore, individual PK parameters were used to calculate the dose of FIX concentrate needed to achieve a steady-state FIX trough activity level of $0.01,0.03,0.05$, and $0.10 \mathrm{IU} / \mathrm{mL}$. 
Table 1 Pharmacokinetic parameters of the population pharmacokinetic models used for simulation

\begin{tabular}{|c|c|c|c|c|}
\hline Parameters & $\mathrm{N} 9-\mathrm{GP}^{\dagger, *}$ & $\mathrm{rFIXFc}^{\ddagger}[15]$ & rIX-FP ${ }^{\S}[16]$ & $\mathrm{rFIX}^{\S}[17]$ \\
\hline $\mathrm{CL}(\mathrm{mL} / \mathrm{h})$ & 0.5101 & 239 & 57 & 560 \\
\hline $\mathrm{V} 1(\mathrm{~mL})$ & 58.9213 & 7140 & 6480 & 6090 \\
\hline Q2 (mL/h) & - & 167 & 29 & 22400 \\
\hline V2 (mL) & - & 8700 & 1580 & 4160 \\
\hline Q3 (ml/h) & - & 3930 & - & 430 \\
\hline V3 (ml) & - & 3990 & - & 3900 \\
\hline \multicolumn{5}{|l|}{ Covariates } \\
\hline Bodyweight effect on CL & - & 0.436 & 0.53 & 0.66 \\
\hline Bodyweight effect on Q2 and Q3 & - & - & - & 0.66 \\
\hline Bodyweight effect on V1 & - & 0.396 & 0.79 & 0.64 \\
\hline Bodyweight effect on V2 & - & - & 0.79 & 0.64 \\
\hline Bodyweight effect on V3 & - & - & - & 0.64 \\
\hline Weight adjusted dose on V1 & - & - & 0.38 & - \\
\hline Age effect on V2 ( $\%$ change with age different from 23 years) & - & - & - & 1.6 \\
\hline \multicolumn{5}{|l|}{ Inter-individual variability (IIV) } \\
\hline IIV on CL (\%) & $16.79^{\mathrm{qI}}$ & 17.7 & 22.6 & 19.0 \\
\hline IIV on V1 $(\%)$ & 14.06 & 21.7 & 26.9 & 46.0 \\
\hline IIV on Q2 (\%) & - & 35.8 & - & - \\
\hline IIV on V2 $(\%)$ & - & 46.2 & - & 37.0 \\
\hline IIV on V3 $(\%)$ & - & 37.7 & - & 28.0 \\
\hline Correlation between IIV Cl and V1 (\%) & - & 75.6 & - & - \\
\hline \multicolumn{5}{|l|}{ Inter-individual variability (IOV) } \\
\hline IOV on CL $(\%)$ & - & 15.1 & - & - \\
\hline IOV on V1 (\%) & - & 17.4 & - & - \\
\hline \multicolumn{5}{|l|}{ Residual variability } \\
\hline Additive error (IU/ml) & 0.01003 & 0.0024 & 0.0066 & 0.0064 \\
\hline Proportional error $(\%)$ & - & 10.6 & 18.0 & 8.7 \\
\hline
\end{tabular}

$C L$ clearance, $V 1$ central volume of distribution, $Q 2$ inter-compartmental clearance of compartment 2, V2 volume of compartment 2, $Q 3$ intercompartmental clearance of compartment $3, V 3$ volume of compartment 3

*Population pharmacokinetic parameters of N9-GP were provided by Novo Nordisk (personal communication)

${ }^{\dagger}$ Parameters scaled to $1 \mathrm{~kg}$

* Parameters CL and V1 scaled to $73 \mathrm{~kg}$ by allometric scaling

$\S$ Parameters scaled to $70 \mathrm{~kg}$ by allometric scaling

${ }^{\text {II }}$ IIV of clearance of N9-GP was taken from Collins [18]

\section{Results}

The distributions of age and bodyweight of the 10,000 virtual patients with severe hemophilia B are depicted in Figure 1. Figure 2 show that the FIX activity level versus time profiles vary between concentrates, demonstrating different PK properties such as exposure and half-life.

For rIX-FP, the longest elimination half-life was obtained (104 h), while the elimination half-lives of N9-GP and rFIXFc were comparable ( 80 and $82 \mathrm{~h}$ ). As expected, these parameters were 4- to 5-fold longer than for the SHL-rFIX concentrate with a value of $22 \mathrm{~h}$ (Table 2). The increase in half-life of the various EHL-FIX concentrates did not result in comparable increases in exposure (AUC). The median AUC of N9-GP $(78.5 \mathrm{IU} / \mathrm{h} / \mathrm{mL})$ was six times higher than the AUC of rFIXFc $(12.1 \mathrm{IU} / \mathrm{h} / \mathrm{mL})$, while the AUC of rIX-FP was four times higher $(49.6 \mathrm{IU} / \mathrm{h} / \mathrm{mL})$ than $\mathrm{rFIXFc}$. This is also reflected in both the calculated trough FIX activity levels which are respectively $0.21,0.14$, and $0.02 \mathrm{IU} / \mathrm{mL}$ for N9GP, rIX-FP, and rFIXFc, and in the time above and below 1, 3,5 , and $10 \mathrm{IU} / \mathrm{mL}$ (Table 2).

Although a weekly dose of $40 \mathrm{IU} / \mathrm{kg}$ produces median FIX activity levels above $0.01 \mathrm{IU} / \mathrm{mL}$ during the complete dosing period of $168 \mathrm{~h}$ ( 1 week) for each of the EHL-FIX concentrate, 
Fig. 1 Distribution of age and bodyweight for the simulated population of 10,000 severe hemophilia B patients

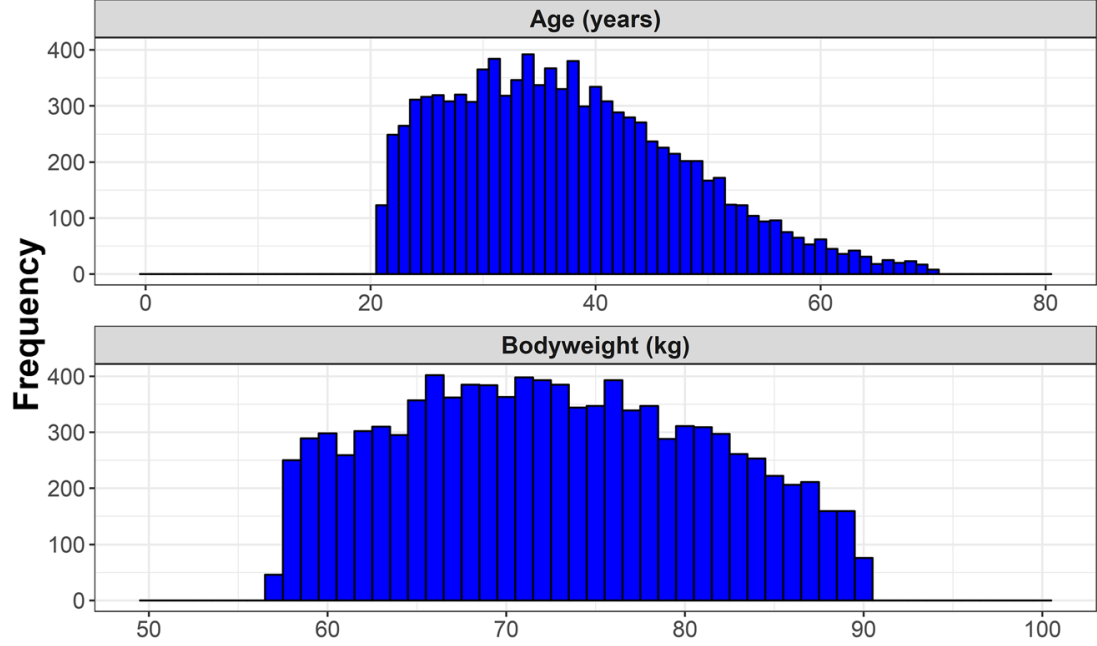

significant differences were observed for a target trough activity level of $0.10 \mathrm{IU} / \mathrm{mL}$. In the latter case, median values for

a

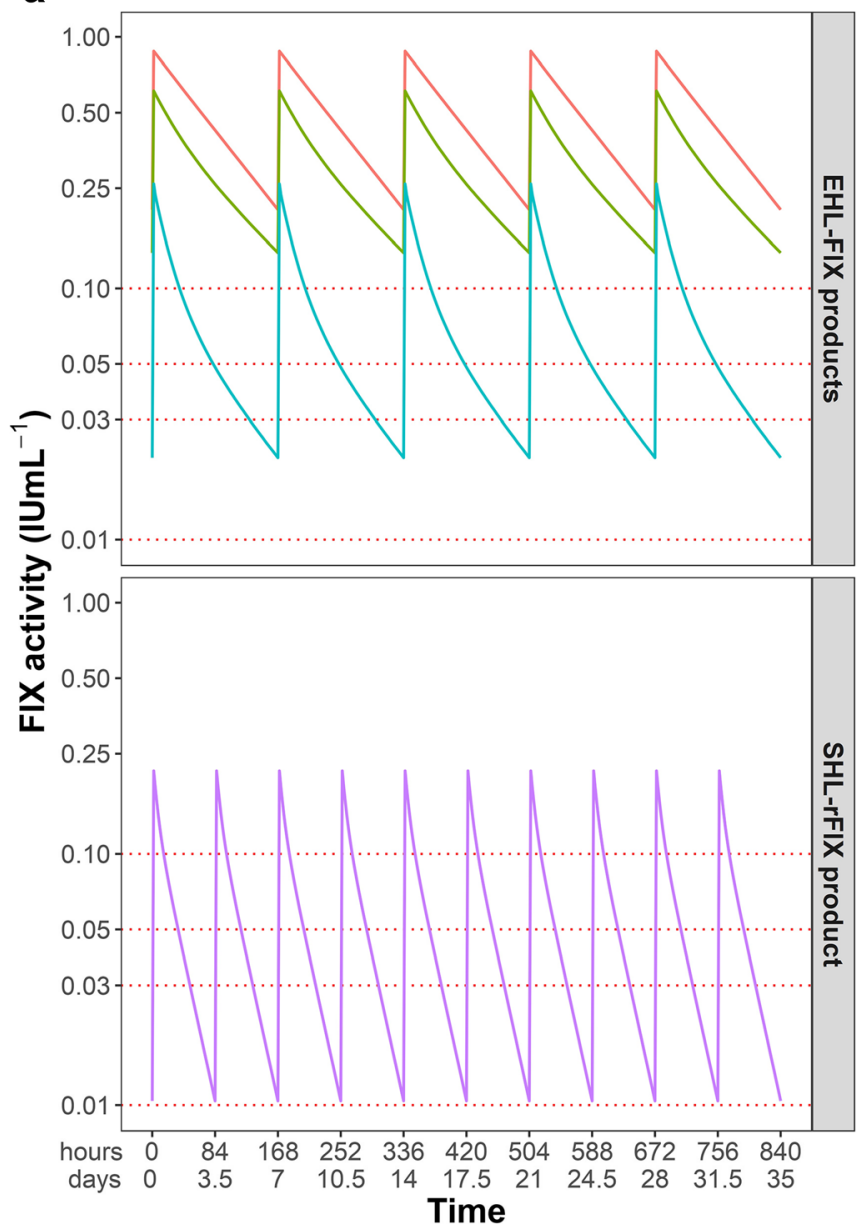

Fig. 2 Simulated FIX activity levels for the examined FIX concentrates. IU, international units. SHL, standard half-life. EHL, extended half-life. a Median FIX activity levels versus time from N9-GP (orange), rIX-FP (green), rFIXFc (blue), and rFIX (purple) for 10,000 patients during steady-state dosing of $40 \mathrm{IU} / \mathrm{kg}$ once weekly (EHL-concentrates) and the time above a target activity level of $10 \mathrm{IU} / \mathrm{mL}$ were respectively 168,168 , and $36 \mathrm{~h}$ for N9-GP, rIX-FP, and rFIXFc.

b

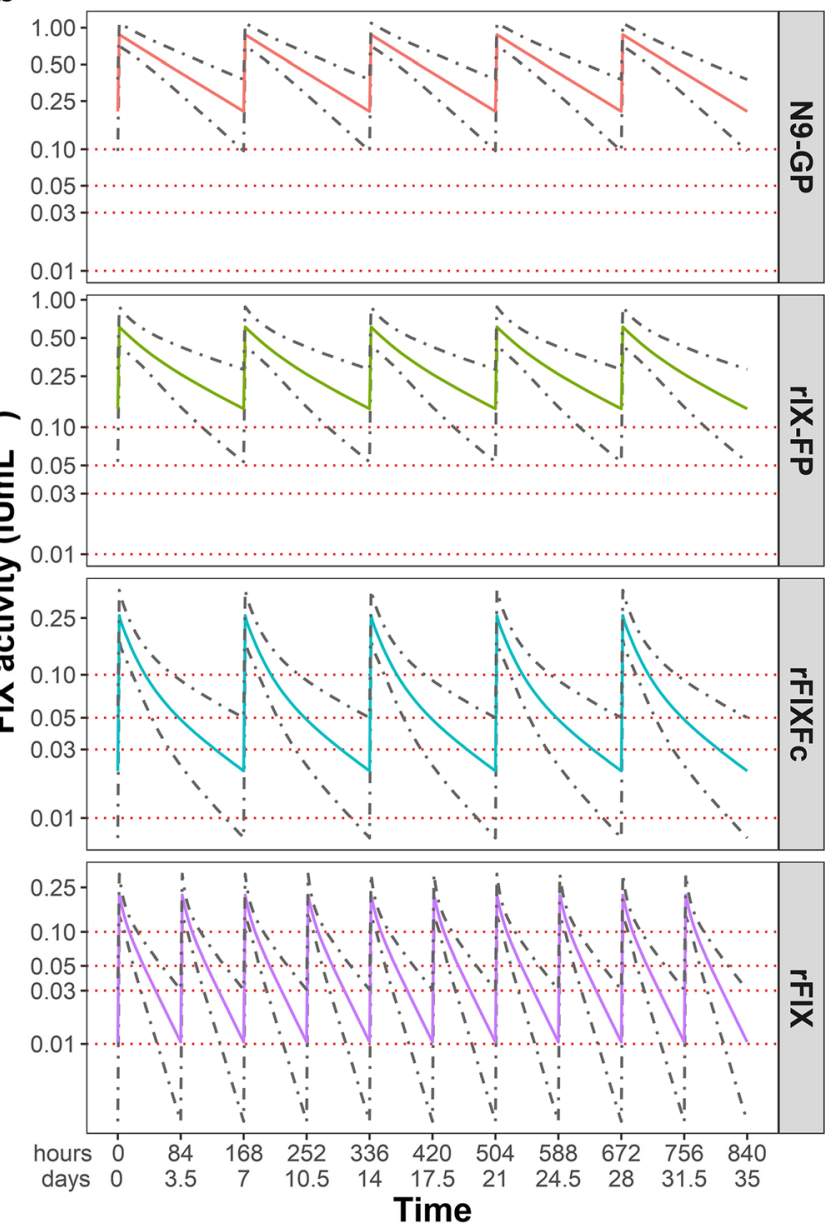

40 IU/kg twice weekly (rFIX). The dashed red lines depict the FIX target trough levels. b Median simulated FIX activity levels from N9-GP (orange), rIX-FP (green), rFIXFc (blue), and rFIX (purple) versus time with the 2.5th and 97.5th percentiles (gray dashed lines) of the FIX activity levels. Note the logarithmically transformed $y$-axis 
Table 2 Simulated pharmacokinetic parameters for steady-state dosing of $40 \mathrm{IU} / \mathrm{kg}$

\begin{tabular}{|c|c|c|c|c|c|c|c|c|}
\hline \multirow[t]{2}{*}{ Parameter } & \multicolumn{2}{|l|}{ N9-GP } & \multicolumn{2}{|l|}{ rIX-FP } & \multicolumn{2}{|l|}{ rFIXFc } & \multicolumn{2}{|l|}{ rFIX } \\
\hline & Median & Range $90 \%$ & Median & Range $90 \%$ & Median & Range $90 \%$ & Median & Range $90 \%$ \\
\hline Terminal elimination half-life (h) & 79.9 & $(56.0-115.1)$ & 104.2 & $(73.6-158.8)$ & 82.2 & $(47.5-158.9)$ & 21.8 & $(14.1-34.5)$ \\
\hline AUC (IU/h/mL) & 78.5 & $(59.3-103.9)$ & 49.6 & $(34.9-71.3)$ & 12.1 & $(8.1-18.2)$ & $10.1^{\dagger}$ & $(7.27-14.0)$ \\
\hline Maximum FIX activity level (IU/mL) & 0.89 & $(0.74-1.08)$ & 0.62 & $(0.46-0.86)$ & 0.42 & $(0.27-0.66)$ & 0.43 & $(0.23-0.80)$ \\
\hline In vivo recovery (IU/dL per IU/kg) & 1.70 & $(1.35-2.15)$ & 1.18 & $(0.78-1.83)$ & 1.00 & $(0.62-1.58)$ & 1.05 & $(0.54-1.99)$ \\
\hline Trough FIX activity level (IU/mL) & 0.21 & $(0.11-0.35)$ & 0.14 & $(0.06-0.26)$ & 0.021 & $(0.009-0.045)$ & 0.010 & $(0.002-0.027)$ \\
\hline Time above $0.01 \mathrm{IU} / \mathrm{mL}(\mathrm{h})$ & 168.0 & $(168.0-168.0)$ & 168.0 & $(168.0-168.0)$ & 168.0 & $(156.1-168.0)$ & $168.0^{\dagger}$ & $(112.2-168.0)$ \\
\hline Time above $0.03 \mathrm{IU} / \mathrm{mL}(\mathrm{h})$ & 168.0 & {$[168.0-168.0)$} & 168.0 & $(168.0-168.0)$ & 129.6 & $(74.5-168)$ & $100.1^{\dagger}$ & $(65.9-157.3)$ \\
\hline Time above $0.05 \mathrm{IU} / \mathrm{mL}(\mathrm{h})$ & 168.0 & $(168.0-168.0)$ & 168.0 & $(168.0-168.0)$ & 80.8 & $(48.0-149.5)$ & $68.8^{\dagger}$ & $(44.8-108.5)$ \\
\hline Time above $0.10 \mathrm{IU} / \mathrm{mL}(\mathrm{h})$ & 168.0 & $(168.0-168.0)$ & 168.0 & $(118.7-168.0)$ & 36.1 & $(21.1-64.6)$ & $30.4^{\dagger}$ & $(19.7-48.6)$ \\
\hline \multicolumn{9}{|l|}{ Dose to achieve target activity } \\
\hline Target trough $0.01 \mathrm{IU} / \mathrm{mL}(\mathrm{IU} / \mathrm{kg})$ & 1.93 & $(1.16-3.68)$ & 2.88 & $(1.55-6.20)$ & 18.9 & $(9.0-46.0)$ & $78.7^{\dagger}$ & $(29.7-33.7)$ \\
\hline Target trough $0.03 \mathrm{IU} / \mathrm{mL}(\mathrm{IU} / \mathrm{kg})$ & 5.80 & $(3.48-11.0)$ & 8.63 & $(4.66-18.6)$ & 56.7 & $(26.9-138.0)$ & $236.1^{\dagger}$ & $(89.0-911.2)$ \\
\hline Target trough $0.05 \mathrm{IU} / \mathrm{mL}(\mathrm{IU} / \mathrm{kg})$ & 9.66 & $(5.80-18.4)$ & 14.4 & $(7.76-31.0)$ & 94.6 & $(44.9-229.8)$ & $393.6^{\dagger}$ & $(148.3-1518)$ \\
\hline Target trough $0.10 \mathrm{IU} / \mathrm{mL}$ (IU/kg) & 19.32 & $(11.6-36.8)$ & 28.8 & $(15.5-62.0)$ & 189.1 & $(89.8-459.7)$ & $787.1^{\dagger}$ & $(296.7-3037)$ \\
\hline
\end{tabular}

The steady-state FIX activity levels of the EHL-FIX concentrates were achieved by dosing $40 \mathrm{IU} / \mathrm{kg}$ every $168 \mathrm{~h}$, whereas steady-state FIX activity levels for rFIX were achieved by dosing $40 \mathrm{IU} / \mathrm{kg}$ every $84 \mathrm{~h}$

$I U$ international units, $A U C$ area under the curve

${ }^{\dagger}$ As rFIX doses were administrated twice weekly; the calculated value depicts the sum of the two doses administered per week

Interestingly, once weekly dosing of $40 \mathrm{IU} / \mathrm{kg} \mathrm{rFIXFc}$ produced similar values for AUC and time above $10 \mathrm{IU} / \mathrm{mL}$ as compared with dosing of rFIX twice weekly. In Table 2, doses to maintain specific target trough activities levels are presented. In comparison with rFIX, the required weekly dose for a target trough activity level of $0.01 \mathrm{IU} / \mathrm{mL}$ was $40-, 27-$, and 4.1-fold lower for N9-GP, rIX-FP, and rFIXFc, respectively. In our study, simulated trough activity level of the EHL-FIX concentrates at $168 \mathrm{~h}$ were in agreement with those clinically observed and reported in literature [15, 16, 18, 25].

In general, after administration of $40 \mathrm{IU} / \mathrm{kg}$, higher peak FIX activity levels were observed for N9-GP and rIX-FP in comparison with rFIXFc. This is also reflected in the calculated in vivo recovery (IVR) values, with N9-GP showing the highest median IVR of $1.70 \mathrm{IU} / \mathrm{dL}$ per IU/kg. rIX-FP, rFIXFc, and rFIX produced lower median IVR values of 1.18, 1.00, and $1.05 \mathrm{IU} / \mathrm{dL}$ per IU $/ \mathrm{kg}$, respectively.

\section{Discussion}

Using Monte Carlo simulations, individual PK parameters and subsequent FIX activity levels over time curves were obtained. The observed terminal half-life values of the EHL-FIX concentrates were comparable, with rIX-FP showing a slightly longer terminal half-life. On the other hand, N9-GP and rIXFP clearly demonstrated higher exposure, higher trough FIX activity levels, longer time above a target level $(0.03,0.05$, or $0.10 \mathrm{IU} / \mathrm{mL}$ ) than rFIXFc. These results are comparable to the PK comparison between N9-GP and rFIX-Fc performed by Escuriola Ettingshausen et al. demonstrating favorable PK for N9-GP [26].

The lower exposure and shorter time above a certain target level of rFIXFc compared with the other EHL-FIX concentrates could indicate that higher rFIXFc doses or shorter dosing intervals are necessary with this concentrate especially for patients that require higher FIX trough levels or patients that require higher FIX activity levels for physical activities. However, it must be taken into account that the characteristic FIX activity level versus time profile of rFIXFc - with a rapid decreasing FIX activity level during the distribution phase and a slower decrease during the elimination phase-is possibly a result of extravascular FIX binding with collagen IV [27]. Just as for rFIX, rFIXFc distribution is not limited to the plasma, and the PK curve displays a rapid distribution to the extravascular compartment $[15,26]$. In comparison, studies have observed that N9-GP mostly remains in plasma compartment, as the PEG moiety of N9-GP possibly reduces distribution to extravascular space $[21,28]$. These differences in distribution are also illustrated by the fact that rFIX and rFIXFc are both described by three compartment models, while the PK of N9-GP and rIX-FP are described by one and two compartment models [15-17]. Several non-clinical studies 
have indicated that extravascular FIX plays a clinically relevant role in hemostasis, but the full extent of this pharmacodynamic effect is yet to be discovered $[27,29,30]$. Although annual bleeding rates (ABR) are not directly comparable, similar median and interquartile ranges of ABR were observed in clinical studies for rFIXFc in adult hemophilia B patients after weekly prophylaxis with similar doses (2.3, IQR 0.44-3.76; median dose $49.5 \mathrm{IU} / \mathrm{kg}$ ) compared with rIX-FP (1.58, IQR: 0.00-4.06; median dose $40.3 \mathrm{IU} / \mathrm{kg})$ and N9-GP (1.04, IQR 0.00-4.00; median dose $40 \mathrm{IU} / \mathrm{kg}$ ) [31-33]. This may indicate that the hemostatic efficacy of rFIXFc is more or less similar despite lower FIX activity levels. As a result, the pharmacodynamic properties ("intrinsic efficacy") of rFIXFc may be different from N9-GP and rIX-FP.

Since this study was performed in silico and used the published population PK models, the results can only be interpreted for a study population similar to the population on which the PK models were originally built. Therefore, the presented results reflect PK parameters for patients from 21 to 65 years and from 57.3 to $90 \mathrm{~kg}$. Furthermore, the blood sampling schemes used for data collection of the population PK models may have influenced the PK properties, as prolonged FIX sampling increases the obtained terminal half-life [34, 35]. Additionally, it is important to realize that varying one-stage assays with varying activators have been applied in population PK studies performed by pharmaceutical companies and in clinical reports, which may additionally contribute to the found differences. Finally, it is important that the presented study results are based on simulations and should be interpreted with caution. Collection of real-world clinical data from patients is still essential, as for instance inter-patient (PK) variability may deviate in the clinical setting. Therefore, it is recommended to perform follow up clinical studies in which concentrates are compared using for instance a cross-over design.

\section{Conclusion}

The simulations in this study show that PK properties of the novel EHL-FIX concentrates differ. Despite the comparable terminal half-lives that were obtained for the investigated EHL-FIX concentrates, different AUCs and different time intervals above a specific FIX activity level were obtained. This study gives insight into specific PK properties of the EHL-FIX concentrates and may therefore support FIX concentrate selection and determination of dosing regimens in the real-life setting of daily hemophilia care. However, to fully unravel the effect of the EHL-FIX concentrates on hemostatic efficacy in hemophilia B, further research exploring the dose and PK-pharmacodynamic relationship is warranted.

Author contribution TP, LB, MS, and RM performed the pharmacokinetic analyses and wrote the manuscript. MC helped design the study and helped with writing the manuscript. FL critically revised the manuscript. All authors approved the final version.

Funding This project was funded by an unrestricted research grant from CSL Behring. Novo Nordisk generously provided population PK information on N9-GP not available in the public domain.

Data Availability For original data, please contact r.mathot@amsterdamumc.nl.

Code availability Please contact r.mathot@amsterdamumc.nl for code sharing.

\section{Declarations}

Conflict of interest FL reports grants from CSL Behring, grants from Shire, grants from uniQure, other from uniQure, other from Shire, other from BioMarin, personal fees from Roche, and outside the submitted work. MC has received grants from governmental research institutes such as Dutch Research Institute (NWO), ZonMW, Innovation fund, NWONWA, and unrestricted investigator initiated research grants as well as educational and travel funding from the following companies over the years: Pfizer, Baxter/Baxalta/Shire, Bayer Schering Pharma, CSL Behring, Sobi Biogen, Novo Nordisk, Novartis and Nordic Pharma, and has served as a member on steering boards of Roche, Bayer, and Octapharma. All grants, awards, and fees go to the institution. RM reports grants from Bayer, grants from Shire, grants from Merck Sharpe Dome, grants from CSL Behring, other from Bayer, and other from Shire outside the submitted work. Other authors declare no competing financial interests.

Open Access This article is licensed under a Creative Commons Attribution 4.0 International License, which permits use, sharing, adaptation, distribution and reproduction in any medium or format, as long as you give appropriate credit to the original author(s) and the source, provide a link to the Creative Commons licence, and indicate if changes were made. The images or other third party material in this article are included in the article's Creative Commons licence, unless indicated otherwise in a credit line to the material. If material is not included in the article's Creative Commons licence and your intended use is not permitted by statutory regulation or exceeds the permitted use, you will need to obtain permission directly from the copyright holder. To view a copy of this licence, visit http://creativecommons.org/licenses/by/4.0/.

\section{References}

1. Franchini M, Frattini F, Crestani S, Bonfanti C (2012) Haemophilia B: Current pharmacotherapy and future directions. Expert Opin Pharmacother 13:2053-2063. https://doi.org/10.1517/14656566. 2012.721780

2. Ahlberg A (1965) Haemophilia in Sweden VII. Incidence, treatment and prophylaxis of arthropathy and other musculo-skeletal manifestations of haemophilia A and B. Acta Orthop Scand Suppl 
36(Suppl 77):3-132. https://doi.org/10.3109/ort.1965.36.suppl-77. 01

3. Fijnvandraat K, Cnossen MH, Leebeek FWG, Peters M (2012) Diagnosis and management of haemophilia. BMJ 344:e2707. https://doi.org/10.1136/bmj.e2707

4. Ahnström J, Berntorp E, Lindvall K, Björkman S (2004) A 6-year follow-up of dosing, coagulation factor levels and bleedings in relation to joint status in the prophylactic treatment of haemophilia. Haemophilia 10:689-697. https://doi.org/10.1111/j.1365-2516. 2004.01036.x

5. Bjorkman S (2003) Prophylactic dosing of factor VIII and factor IX from a clinical pharmacokinetic perspective. Haemophilia 9:101110. https://doi.org/10.1046/j.1365-2516.9.s1.4.x

6. Collins PW, Blanchette VS, Fischer K et al (2009) Break-through bleeding in relation to predicted factor VIII levels in patients receiving prophylactic treatment for severe hemophilia A. J Thromb Haemost 7:413-420. https://doi.org/10.1111/j.1538-7836.2008. 03270.x

7. Den Uijl IEM, Fischer K, Van Der Bom JG et al (2011) Analysis of low frequency bleeding data: the association of joint bleeds according to baseline FVIII activity levels. Haemophilia 17:41-44. https:// doi.org/10.1111/j.1365-2516.2010.02383.x

8. Iorio $\mathrm{A}$, Iserman $\mathrm{E}$, Blanchette $\mathrm{V}$, Dolan $\mathrm{G}$, Escuriola Ettingshausen C, Hermans C, Negrier C, Oldenburg J, Reininger A, Rodriguez-Merchan C, Spannagl M, Valentino LA, Young G, Steinitz-Trost KN, Gringeri A (2017) Target plasma factor levels for personalized treatment in haemophilia: a Delphi consensus statement. Haemophilia 23:e170-e179. https://doi.org/10.1111/ hae. 13215

9. Mancuso M, Santagostino E (2017) Outcome of clinical trials with new extended half-life FVIII/IX concentrates. J Clin Med 6:39. https://doi.org/10.3390/jcm6040039

10. Graf L (2018) Extended half-life factor VIII and factor IX preparations. Transfus. Med. Hemotherapy 45:86-91. https://doi.org/10. $1159 / 000488060$

11. Taylor JA, Kruse-Jarres R (2016) A new era for hemophilia B treatment. Blood 127:1734-1736. https://doi.org/10.1182/blood2016-02-694869

12. Nazeef M, Sheehan JP (2016) New developments in the management of moderate-to-severe hemophilia B. J Blood Med 7:27-38. https://doi.org/10.2147/JBM.S81520

13. Collins P, Chalmers E, Chowdary P, Keeling D, Mathias M, O'Donnell J, Pasi KJ, Rangarajan S, Thomas A (2016) The use of enhanced half-life coagulation factor concentrates in routine clinical practice: guidance from UKHCDO. Haemophilia 22:487-498. https://doi.org/10.1111/hae.13013

14. Bonate PL (2001) A brief introduction to Monte Carlo simulation. Clin Pharmacokinet 40:15-22. https://doi.org/10.2165/00003088200140010-00002

15. Diao L, Li S, Ludden T, Gobburu J, Nestorov I, Jiang H (2014) Population pharmacokinetic modelling of recombinant factor IX Fc fusion protein $(\mathrm{rFIXFc})$ in patients with haemophilia B. Clin Pharmacokinet 53:467-477. https://doi.org/10.1007/s40262-0130129-7

16. Zhang Y, Roberts J, Bensen-Kennedy D, Jacobs I, Santagostino E, Voigt C, Feussner A, Morfini M, Sidhu J (2016) Population pharmacokinetics of a new long-acting recombinant coagulation factor IX albumin fusion protein for patients with severe hemophilia B. J Thromb Haemost 14:2132-2140. https://doi.org/10.1111/jth.13444

17. Björkman S (2013) Population pharmacokinetics of recombinant factor IX: Implications for dose tailoring. Haemophilia 19:753757. https://doi.org/10.1111/hae.12188

18. Collins PW, Møss J, Knobe K et al (2012) Population pharmacokinetic modeling for dose setting of nonacog beta pegol (N9-GP), a glycoPEGylated recombinant factor IX. J Thromb Haemost 10: 2305-2312. https://doi.org/10.1111/jth.12000
19. Negrier C, Knobe K, Tiede A, Giangrande P, Møss J (2011) Enhanced pharmacokinetic properties of a glycoPEGylated recombinant factor IX: A first human dose trial in patients with hemophilia B. Blood 118:2695-2701. https://doi.org/10.1182/blood2011-02-335596

20. Oldenburg J, Carcao M, Lentz SR, Mahlangu J, Mancuso ME, Matsushita T, Négrier C, Clausen WHO, Ehrenforth S, Young G (2018) Once-weekly prophylaxis with $40 \mathrm{IU} / \mathrm{kg}$ nonacog beta pegol (N9-GP) achieves trough levels of $>15 \%$ in patients with haemophilia B: Pooled data from the paradigm ${ }^{\mathrm{TM}}$ trials. Haemophilia 24:911-920. https://doi.org/10.1111/hae.13608

21. Tiede A, Abdul-Karim F, Carcao M, Persson P, Clausen WHO, Kearney S, Matsushita T, Negrier C, Oldenburg J, Santagostino E, Young G (2017) Pharmacokinetics of a novel extended halflife glycoPEGylated factor IX, nonacog beta pegol (N9-GP) in previously treated patients with haemophilia B: results from two phase 3 clinical trials. Haemophilia 23:547-555. https://doi.org/ 10.1111/hae.13191

22. Rosén P, Rosén S, Ezban M, Persson E (2016) Overestimation of $\mathrm{N}$-glycoPEGylated factor IX activity in a one-stage factor IX clotting assay owing to silica-mediated premature conversion to activated factor IX. J Thromb Haemost 14:1420-1427. https://doi. org/10.1111/jth.13359

23. Bowyer AE, Hillarp A, Ezban M, Persson P, Kitchen S (2016) Measuring factor IX activity of nonacog beta pegol with commercially available one-stage clotting and chromogenic assay kits: a two-center study. J Thromb Haemost 14:1428-1435. https://doi. org/10.1111/jth. 13348

24. Core Team R (2018) R: A language and environment for statistical computing. R Foundation for Statistical Computing, Vienna

25. Young G, Collins PW, Colberg T, Chuansumrit A, Hanabusa H, Lentz SR, Mahlangu J, Mauser-Bunschoten EP, Négrier C, Oldenburg J, Patiroglu T, Santagostino E, Tehranchi R, Zak M, Karim FA (2016) Nonacog beta pegol (N9-GP) in haemophilia B: a multinational phase III safety and efficacy extension trial (paradigm $^{\text {TM }} 4$ ). Thromb Res 141:69-76. https://doi.org/10.1016/j. thromres.2016.02.030

26. Escuriola Ettingshausen C, Hegemann I, Simpson ML, Cuker A, Kulkarni R, Pruthi RK, Garly ML, Meldgaard RM, Persson P, Klamroth R (2019) Favorable pharmacokinetics in hemophilia B for nonacog beta pegol versus recombinant factor IX-Fc fusion protein: a randomized trial. Res Pract Thromb Haemost 3:268276. https://doi.org/10.1002/rth2.12192

27. Stafford DW (2016) Extravascular FIX and coagulation. Thromb J 14:35. https://doi.org/10.1186/s12959-016-0104-2

28. Salas J, van der Flier A, Hong V et al (2017) Extravascular distribution of conventional and Ehl FIX products using in vivo SPECT imaging analysis in hemophilia B mice. Blood 130(Supplement 1): 1061

29. Feng D, Stafford KA, Broze GJ, Stafford DW (2013) Evidence of clinically significant extravascular stores of factor IX. J Thromb Haemost 11:2176-2178. https://doi.org/10.1038/jid.2014.371

30. Cooley B, Funkhouser W, Monroe D, Ezzell A, Mann DM, Lin FC, Monahan PE, Stafford DW (2016) Prophylactic efficacy of BeneFIX vs Alprolix in hemophilia B mice. Blood 128:286-292. https://doi.org/10.1182/blood-2016-01-696104

31. Pasi KJ, Fischer K, Ragni M, Nolan B, Perry DJ, Kulkarni R, Ozelo M, Mahlangu J, Shapiro AD, Baker RI, Bennett CM, Barnes C, Oldenburg J, Matsushita T, Yuan H, Ramirez-Santiago A, Pierce GF, Allen G, Mei B (2017) Long-term safety and efficacy of extended-interval prophylaxis with recombinant factor IX Fc fusion protein $(\mathrm{rFIXFc})$ in subjects with haemophilia B. Thromb Haemost 117:508-518. https://doi.org/10.1160/TH16-05-0398

32. Santagostino E, Martinowitz U, Lissitchkov T et al (2016) Longacting recombinant coagulation factor IX albumin fusion protein 
(rIX-FP) in hemophilia B: results of a phase 3 trial. Blood 127: 1761-1769. https://doi.org/10.1160/TH16-03-0179

33. Collins PW, Young G, Knobe K, Karim FA, Angchaisuksiri P, Banner C, Gürsel T, Mahlangu J, Matsushita T, MauserBunschoten EP, Oldenburg J, Walsh CE, Negrier C, paradigm 2 Investigators (2014) Recombinant long-acting glycoPEGylated factor IX in hemophilia B: a multinational randomized phase 3 trial. Blood 124:3880-3886. https://doi.org/10.1182/blood-2014-05573055

34. Hua B, Wu R, Sun FF, Luo B, Alvey C, LaBadie RR, Qu PR, Korth-Bradley JM, Rendo P (2017) Confirmation of longer FIX activity half-life with prolonged sample collection after single doses of nonacog alfa in patients with haemophilia B. Thromb Haemost 117:1052-1057. https://doi.org/10.1160/TH16-10-0765
35. Mahlangu J, Powell JS, Ragni MV, Chowdary P, Josephson NC, Pabinger I, Hanabusa H, Gupta N, Kulkarni R, Fogarty P, Perry D, Shapiro A, Pasi KJ, Apte S, Nestorov I, Jiang H, Li S, Neelakantan S, Cristiano LM, Goyal J, Sommer JM, Dumont JA, Dodd N, Nugent K, Vigliani G, Luk A, Brennan A, Pierce GF, A-LONG Investigators (2014) Phase 3 study of recombinant factor VIII Fc fusion protein in severe hemophilia A. Blood 123:317-325. https:// doi.org/10.1182/blood-2013-10-529974

Publisher's note Springer Nature remains neutral with regard to jurisdictional claims in published maps and institutional affiliations. 